(2) Open Access Full Text Article

\title{
Assessment of risk and prophylaxis for deep vein thrombosis and pulmonary embolism in medically ill patients during their early days of hospital stay at a tertiary care center in a developing country
}

This article was published in the following Dove Press journal:

Vascular Health and Risk Management

4 August 2009

Number of times this article has been viewed

\section{Ambarish Pandey \\ Nivedita Patni \\ Mansher Singh \\ Randeep Guleria}

Department of Medicine, All India Institute of Medical Sciences, New Delhi, India
Correspondence: Randeep Guleria Department of Internal Medicine, All India Institute of Medical Sciences, Ansari Nagar, New Delhi, India I 10029 Tel +9 I I I 26593676

Email randeepg@hotmail.com
Aim: Deep vein thrombosis (DVT) and pulmonary thromboembolism (PE) are important causes of morbidity and mortality in medically ill patients. This study was done to assess risk factors and prophylaxis given for DVT and PE in newly admitted medically ill patients during the first two weeks of their hospital stay at a tertiary care center hospital in India.

Methods: All patients within one week of their admission in intensive care unit (ICU) and wards were enrolled in the study after an informed written consent. Patients who had DVT prophylaxis within the past month or any contraindications for DVT prophylaxis were excluded. A structured proforma was designed and effective risk stratification for DVT was done. Patients were followed for up to two weeks to record any changes in the risk categories and document any signs of PE or DVT if present. Any prophylaxis given for DVT or PE was noted.

Results: Seventy-five percent of patients had the highest risk for DVT and PE. Only 12.5\% had DVT prophylaxis within the first two days of admission. Within two weeks of admission, $30.8 \%$ of patients were discharged, and $16.2 \%$ died. $72.6 \%$ of the patients still in the wards belonged to the highest risk category. Clinical signs and symptoms of DVT and PE were present in $25.8 \%$ and $9.8 \%$ of patients, respectively after the second week of admission. $86 \%$ of symptomatic patients belonged to the highest risk category initially and none of them received any prophylaxis. $21.6 \%$ of the highest risk category patients died within two weeks of their admission. A statistically significant correlation was found between mortality and risk score of the patients for DVT and between lack of prophylaxis and mortality $(\mathrm{p}<0.05)$.

Conclusion: A significant risk for DVT and PE exists in medically ill patients, but only a small proportion of the patients are given prophylaxis. This study underlines the need to aggressively implement DVT risk stratification strategy in medical patients and provide prophylaxis unless contraindicated.

Keywords: deep venous thrombosis, pulmonary embolism, risk stratification, DVT prophylaxis

\section{Introduction}

Venous thromboembolism (VTE), which includes deep vein thrombosis (DVT) and pulmonary embolism (PE), is an important cause of morbidity and mortality in critically ill patients. ${ }^{1}$ Incidence of symptomatic DVT or PE or any other complication related to venous thromboembolism in critically ill patients not receiving any prophylaxis is $3.6 \%$ in western hospitals and it contributes significantly to the health care cost. ${ }^{2}$

In developing countries such as India, a significant prevalence of etiological risk factors for DVT and prothrombotic factors has been shown amongst hospitalized patients. ${ }^{3}$ 
Studies have shown a need of DVT prophylaxis in $95 \%$ of intensive care unit (ICU) patients in India with significant underuse of prophylaxis in only $55 \%$ of the high risk patients. ${ }^{4}$ Another study in Indian population has shown a overall incidence of confirm DVTs to be 17.46 per hundred thousand patients with $64 \%$ being nonsurgical nontrauma patients. $^{5}$

Critically ill patients are at increased risk of VTE due to predisposing premorbid conditions, occurrence of sepsis, trauma, and post-admission events. ${ }^{6}$ Individual identification of suspected DVT and PE cases could be a difficult task and many cases could be missed. However, blanket prophylaxis of all admitted patients may not be cost-effective, especially in a developing country such as India. ${ }^{7}$ Thus primary prevention of VTE with risk assessment and stratification for DVT and subsequent antithrombotic prophylaxis in moderate to severe risk category patients is the most rational means of reducing mortality and morbidity.

The American College of Chest Physicians (ACCP) developed guidelines for the use of low molecular weight heparins (LMWH) and unfractionated heparins (UFH) in the prevention of VTEs in patients with acute illnesses. ${ }^{8}$ However the use of DVT prophylaxis in hospitalized medical patients still remains suboptimal, around 15\%-16\%. ${ }^{9}$

This is an observational study done to evaluate and assess the risk factors and prophylaxis given for DVT and $\mathrm{PE}$ in patients admitted in medical wards and ICU during the first two weeks of their hospital stay using Caprini's risk stratification score card ${ }^{10}$ at a tertiary care center hospital in India. We looked into the prevalence of risk factors for DVT and PE in these patients within one week of their admission and the interventions done by health care professionals to prevent their occurrence in these patients.

\section{Methods}

This is a prospective observational study done in the medicine ICU and wards at the All India Institute of Medical Sciences, a tertiary health care center in north India. The study did not affect patient care. Health care providers were not aware that this study was being conducted. All patients within one week of their admission in the medicine ward or ICU were enrolled in the study after written informed consent. Patients who had been on DVT prophylaxis within one month of their admission and those who had absolute or relative contraindications for DVT prophylaxis such as gastrointestinal bleeding, history of intracranial bleed, severe bleeding disorder, severe thrombocytopenia, recent neurosurgery, or liver cirrhosis were excluded from the study. A structured proforma was designed for risk assessment and stratification of DVT in critically ill patients using a previously published standard protocol (Caprini's risk stratification score card). ${ }^{10}$ The risk factors for DVT used in this protocol to stratify patients are similar to that published by ACCP and the International Union of Angiology (IUAS) consensus statement and has been used previously for DVT risk assessment alongside ACCP and IUAS risk score models in other studies done in developing countries. ${ }^{11}$ The risk assessment and stratification scorecard used is shown in Figure 1. Demographic data including patient's age, sex, and body weight were collected. Other baseline information like admitting diagnosis, any invasive instrumentation like ventilator, venous catheters, etc were also noted down. Effective risk stratification for DVT was done in low, moderate, high, and highest categories according to the patients DVT risk score at their time of admission as shown in Figure 1.

Patients were followed up for over two weeks from their day of admission. Any change in the patients' general morbidity status and their DVT risk profile was recorded. Patients were examined for any clinical signs and symptoms of DVT or PE (Table 1). Any prophylaxis given and relevant investigations for DVT (D-dimer, Doppler ultrasound, high resolution chest computed tomography [CT], pulmonary angiography) that was done during this time interval was also noted. The data was analyzed using SPSS software (v. 12; SPSS Inc., Chicago, IL, USA).

\section{Results}

One hundred fifty patients were approached for our study within one week of their admission. Of these, $13(8.67 \%)$ patients refused consent, $16(10.67 \%)$ had one or more contraindications for DVT, $4(2.67 \%)$ had DVT prophylaxis in the past three months. Finally, 117 patients were enrolled in the study. Of these, $68(58.11 \%)$ were in the wards and $49(41.89 \%)$ were in the ICU. Table 2 shows the demographic distribution of the patients in the study. Baseline clinical characteristics of the patients are shown in Table 3. We observed that 75\% (100\% ICU; 57.5\% ward) of patients on their initial visit had the highest risk for DVT and PE according to the Caprini's risk stratification score card. ${ }^{10}$ Detailed risk category distribution of the patients during the first visit is shown in Table 4. Respiratory system involvement was seen in $41.8 \%$ of patients while sepsis and septicemia or other infectious etiologies were seen in 
A (each item represents one risk score)

- Minor surgery planned

- Age 41-60 years

- History of prior major surgery $<1$ month

- Pregnancy or postpartum <1 month

- Varicose veins

- IBD

- OCP/HRT

- Obesity

- Swollen legs

C (each item represents three risk scores)

- History of DVT

- MI

- $\mathrm{H} / \mathrm{O}$ thrombosis

- Age $>75$ years

- $\mathrm{CHF}$

- COPD

- Severe sepsis or infection

- Abnormal pulmonary function

- Respiratory assistance

- Myeloproliferative disorder

- Congential or acquired thrombophilia
B (each item represents two risk scores)

- Age >60 years

- Malignancy or chemotherapy

- Immobilized plaster cast

- Bed ridden for $>72$ hours

- Central venous accesses

\author{
D (each item represents five risk scores) \\ - Stroke \\ - Multiple trauma \\ - Acute spinal injury
}

\begin{tabular}{|cc|}
\hline $\begin{array}{c}\text { Total score } \\
0\end{array}$ & $\begin{array}{c}\text { Risk category } \\
\text { No risk }\end{array}$ \\
1 & Low risk \\
2 & Moderate risk \\
$3-4$ & High risk \\
$>5$ & Highest risk \\
\hline
\end{tabular}

Figure I Score card used for effective DVT risk stratification of the subjects.

Abbreviations: CHF, chronic heart failure; COPD, chronic obstructive pulmonary disease; DVT, deep vein thrombosis; HRT, hormone replacement therapy; IBD, irritable bowel disease; $\mathrm{OCP}$, oral contraceptive pill.

$25.6 \%$ of patients. Cardiovascular system involvement was seen in $17.1 \%$ of patients. Multiple system involvement was seen in many patients. Primary respiratory system involvement was found in $35.5 \%$ of patients who had highest risk for DVT. Sepsis and septicemia was seen in $25.6 \%$ of patients with highest risk for DVT. Cardiovascular system involvement was seen in $16.2 \%$ of cases with the highest risk for DVT.

Table I Signs and symptoms of DVT and PE that were looked for in the subject patients

Signs and symptoms of DVT

- Pain or tenderness in the leg

- Swelling of the leg or along a vein in the leg

- Red or discolored skin on the leg

- Increased warmth in the area of the leg that's swollen or is in pain

Signs and symptoms of PE

- Unexplained shortness of breath

- Pain with deep breathing

- Coughing up blood

- Rapid breathing and fast heart rate

Abbreviations: DVT, deep vein thrombosis; $\mathrm{PE}$, pulmonary embolism.
On follow up $16.2 \%$ (22.4\% ICU; $11.7 \%$ ward) of patients died and 30.7\% (14.3\% ICU; 42.6\% ward) of patients got discharged within two weeks of their admission. Of the remaining 62 patients that were still in hospital after two weeks of admission, $72 \%$ (96.6\% ICU; $48.8 \%$ ward) of patients had the highest risk for DVT and PE. $90 \%$ of patients who had highest risk for the DVT and PE after two weeks initially also belonged to the highest risk category. Thus only a minor fraction, $10 \%$ of admitted patients, had progressive increase in their risk scoring for DVT. Two or more signs and symptoms of DVT and PE were seen in $35.5 \%$ (22 of 62) of the patients that were still in hospital after two weeks in hospital. $86.6 \%$ (19 of 22) of these symptomatic patients initially belonged to the highest risk category.

DVT prophylaxis was started within first two days of admission in only $12.5 \%$ (11 of 88 ) of patients who belonged to the highest risk category for DVT and PE initially. None of the patients belonging to moderate or high risk categories received any prophylaxis. All the DVT prophylaxis administered was in form of LMWH. Pneumatic compressions or electric stockings or any other form of mechanical 
Table 2 Demographic details of the patients

\begin{tabular}{ll}
\hline Total no. of patients & 1 I 7 \\
No. of ward patients & $68(58.1$ I\%) \\
No. of ICU patients & $49(41.89 \%)$ \\
No. of males & $72(61.5 \%)$ \\
No. of females & $45(38.5 \%)$ \\
Mean age & $43.7 \pm 19.46$ \\
Mean weight & $53.6 \pm 23.42$
\end{tabular}

Abbreviation: ICU, intensive care unit.

DVT prophylaxis were not used in any patients. No major complications from DVT prophylaxis such as severe bleeding or thrombocytopenia developed in any of the patients who received anticoagulation. None of the symptomatic patients had received any prophylaxis for DVT during course of their admission. 59.1\% $(n=13)$ of the symptomatic patients had one or more relevant investigations for DVT or PE. These investigations included D-dimer levels, ultrasound Doppler of the lower limbs, high resolution CT scan of the chest. $22.7 \%(n=5)$ of the symptomatic patients for DVT or PE were documented to have DVT (three by Doppler ultrasound) or PE (two by high resolution CT scan) by investigators. $21.6 \%(n=19)$ of the highest risk category patients died within one week of follow-up and none of these patients received any prophylaxis for DVT. A statistically significant correlation was found between the mortality and risk scoring of the patients $(\mathrm{p}<0.05)$.

Table 3 Baseline clinical characteristics of the patients

\begin{tabular}{ll}
\hline Baseline characteristics & No. of subjects (\%) \\
\hline Primary etiology & $49(41.8 \%)$ \\
- Respiratory & $30(25.6 \%)$ \\
- Sepsis, septicemia, and other & \\
infectious disease & $20(17.1 \%)$ \\
- Cardiovascular & $20(17.1 \%)$ \\
- Diabetes & $8(6.8 \%)$ \\
- Cancer & $17(14.5 \%)$ \\
- Others & $66(56.4 \%)$ \\
Invasive instrumentations & $98 \pm 18$ \\
Mean heart rate & $123.3 \pm 17.6 \mathrm{mmHg}$ \\
Mean systolic blood pressure & $78.8 \pm 12.2 \mathrm{mmHg}$ \\
Mean diastolic blood pressure & \\
Sensorium & $71(60.7 \%)$ \\
Conscious & $36(30.8 \%)$ \\
Unconscious & $10(8.5 \%)$ \\
\hline Altered sensorium &
\end{tabular}

Notes: *More than one system involvement was seen in many patients.
Table 4 Risk categorization of the patients of DVT risk during their initial visit

\begin{tabular}{lll}
\hline & Ward $(\mathrm{n}=68)$ & ICU $(\mathrm{n}=49)$ \\
\hline No risk & $6(8.8 \%)$ & $0(0 \%)$ \\
Low risk & $6(8.8 \%)$ & $0(0 \%)$ \\
Moderate risk & $8(11.7 \%)$ & $0(0 \%)$ \\
High risk & $9(13.2 \%)$ & $0(0 \%)$ \\
Highest risk & $39(57.5 \%)$ & $49(100 \%)$ \\
\hline
\end{tabular}

Abbreviations: DVT, deep vein thrombosis; ICU, intensive care unit.

Also we found a statistically significant correlation between lack of DVT $(\mathrm{p}<0.05)$ prophylaxis and mortality in our subjects.

\section{Discussion}

The study shows that a significant percentage of admissions in medical wards and ICU are subject to very high risk of DVT and PE starting from the early days of hospital stay. However there is a serious lack of effective prophylaxis for the same in these patients. Our study found that only a minor fraction of the patients who belonged to highest risk category got prophylaxis for DVT.

In our health care set up DVT has traditionally been recognized as a complication of long-term hospitalization in medically ill patients and is often ignored in the initial few days of admission. Similar results have been shown in past studies done in India and other developing countries. In Brazil, DVT prophylaxis risk assessment using Caprini's score card also showed a significant underutilization of DVT prophylaxis. ${ }^{11}$ Our study highlights that a significant proportion of patients have increased risks for DVT even during the first few days of admission, which, if overlooked, could be responsible for life-threatening complications at the later stages. Early recognition of these risk factors and prompt prophylaxis in high-risk cases can be really effective in preventing these life-threatening complications. ACCP has laid down clear guidelines on DVT prophylaxis in medically ill patients. ${ }^{8}$ We found a lack of implementation of any DVT prophylaxis strategy among acutely ill patients in the first two weeks of their management in our institution with only $\sim 11 \%$ of the highest risk patients getting DVT prophylaxis. None of the moderate to high risk patients in our study got any prophylaxis for VTE. Several studies done in the West have also shown an underuse of DVT prophylaxis in hospitalized medical patients despite ACCP recommendations. ${ }^{8,9,12-14}$ 
A survey of health care professionals in the West has shown that almost all of them recognize the importance of DVT prophylaxis, however only half of them implement current DVT prophylaxis strategies in real practice. ${ }^{15}$ Reasons that could play a role include concerns about an increased risk for bleeding from anticoagulants, lack of clear indications and contraindications for anticoagulant prophylaxis, and lack of time to consider VTE prophylaxis in every patient. ${ }^{16}$ Lack of awareness and concerns for cost of DVT prophylaxis in all critically ill patients could also be an important factor, particularly in developing countries such as India. We could not locate any literature to throw light on the reasons for lack of DVT prophylaxis in developing countries such as India and considerable work needs to be done to find out the actual hindrances in this regard.

In our study, a significant proportion of highest risk category patients $(21.6 \%)$ died within their first two weeks of stay in hospital. Though the cause of death was not further looked into to document a confirmed DVT/PE, we clearly saw a statistically significant relation in mortality and high-risk predisposition for DVT. Detailed meta-analysis of all clinical trials to asses the efficacy of DVT prophylaxis in preventing adverse events like DVT/PE have shown a significant reduction (57\% risk reduction) in risk for any PE. ${ }^{17}$ Thus clearly implementation of DVT prophylaxis strategies is the way to go to decrease the mortality and morbidity caused by events like PE in critically ill patients.

Various strategies have been used to increase VTE prophylaxis rates in medically ill patients. Studies have been done that show increase in DVT prophylaxis with strategies such as conducting educational programs to increase awareness for DVT prophylaxis ${ }^{18,19}$ and a computerized alert program for risk assessment and prophylaxis recommendation. ${ }^{20}$

Formulation and implementation of locally developed clinical guidelines has been shown to be beneficial in increasing the VTE prophylaxis rate and bringing down the rate of VTE episodes in individual hospital settings, ${ }^{21}$ and in general health care systems of other countries such as Italy. ${ }^{22}$ Presently we do not have any formalized guidelines, standardized order sets, alerting programs, training, or risk-stratification tools being used at our institution. This study highlights the importance of formulation of locally adapted clinical guidelines and their implementation in initial management of patients. Incorporation of risk assessment and stratification score card for acutely ill patients as a part of their initial management as done in this study could be a simple and cost-effective way of identifying patients for DVT prophylaxis.

\section{Limitations}

Our small sample size of patients could be a limiting factor in predicting an actual prevalence of DVT and PE risk in acutely ill patients in our settings. No investigations were performed to document cases of clinically silent DVT. We did not follow up the patients after discharge or after two weeks of stay in hospital. Of the patients who died, we had not inquired into specific causes during their hospital stay so could not document PE as the cause of death.

\section{Conclusion}

A significant risk for DVT and PE exists in acutely ill patients admitted to wards and ICU, but only a small proportion of the patients are currently given prophylaxis or investigated. This may be responsible for the increased mortality and poor prognosis seen in patients with the highest risk for DVT and PE. This study underlines the need to aggressively implement DVT risk stratification strategy in medical patients and provide prophylaxis unless contraindicated. Successful programs like formulation of DVT prophylaxis guidelines, incorporation of risk assessment and stratification tools in routine patient workup, and interventions to increase awareness of DVT prophylaxis in health care professionals need to be implemented in our heath care system to improve VTE prophylaxis and decrease rates of VTE.

\section{Disclosure}

Ambarish Pandey and Nivedita Patni contributed equally in the study and were involved in literature review, study design, data collection, analysis, and manuscript writing. Mansher Singh contributed to data collection and data analysis. Senior author for this study, Randeep Guleria, was responsible for study design and supervision of data collection analysis and manuscript writing. The authors report no conflicts of interest in this work.

\section{References}

1. Alikhan R, Peters F, Wilmott R, Cohen AT. Fatal pulmonary embolism in hospitalised patients: a necropsy review. J Clin Pathol. 2004;57:1254-1257.

2. Kanaan AO, Silva MA, Donovan JL, Roy T, Al-Homsi AS Meta-analysis of venous thromboembolism prophylaxis in medically III patients. Clin Ther. 2007;29:2395-2405.

3. Garewal G, Das R, Ahluwalia S, Mittal N, Varma S. Prevalence of risk factors for VTE: A study from north India [abstract]. Journal of Thrombosis and Hemostasis. 2005;3(Suppl 1):P1270.

4. Ansari K, Dalal K, Patel M. Risk stratification and utilisation of thrombo-embolism prophylaxis in a medical-surgical ICU: a hospitalbased study. J Indian Med Assoc. 2007;105:536-540.

5. Lee AD, Stephen E, Agarwal S, Premkumar P. Venous thromboembolism in India. Eur J Vasc Endovasc Surg. 2009;37(4):482-485. 
6. Attia J, Ray JG, Cook DJ, Douketis J, Ginsberg JS, Geerts W. Prophylaxis of venous thromboembolism in the critically ill. Arch Intern Med. 2001;161:1268-1279.

7. Nuijten MJ, Berto P, Kosa J, et al. Cost-effectiveness of enoxaparin as thromboprophylaxis in acutely ill medical patients from the Italian NHS perspective. Recent Prog Med. 2002;93:80-91.

8. Geerts WH, Pineo GF, Heit JA, et al. Prevention of venous thromboembolism: the Seventh ACCP Conference on Antithrombotic and Thrombolytic Therapy. Chest. 2004;126(3 Suppl):338S-400S.

9. Kahn SR, Panju A, Geerts W, et al. Multicenter evaluation of the use of venous thromboembolism prophylaxis in acutely ill medical patients in Canada. Thromb Res. 2007;119:145-155.

10. Caprini JA, Arcelus JI, Reyna JJ. Effective risk stratification of surgical and nonsurgical patients for venous thromboembolic disease. Semin Hematol. 2001;38(2 Suppl 5):12-19.

11. Deheinzelin D, Braga AL, Martins LC, et al. Incorrect use of thromboprophylaxis for venous thromboembolism in medical and surgical patients: results of a multicentric, observational and cross-sectional study in Brazil. J Thromb Haemost. 2006;4:1266-1270.

12. Goldhaber SZ, Tapson VF; DVT FREE Steering Committee. A prospective registry of 5,451 patients with ultrasound-confirmed deep vein thrombosis. Am J Cardiol. 2004;93:259-262.

13. Tapson VF, Decousus H, Bergmann JF, et al. The International Medical Prevention Registry on Venous Thromboembolism (IMPROVE): Venous thromboembolism prophylaxis practices in acutely ill medical patients [abstract]. Blood. 2004;104:11, \#1762.

14. Monreal M, Kakkar AK, Caprini JA, et al. The outcome after treatment of venous thromboembolism is different in surgical and acutely ill medical patients. Findings from the RIETE registry. J Thromb Haemost. 2004;2:1892-1898.
15. Kakkar AK, Davidson BL, Haas SK. Compliance with recommended prophylaxis for venous thromboembolism: improving the use and rate of uptake of clinical practice guidelines. J Thromb Haemost. 2004;2:221-227.

16. Douketis JD, Moinuddin I. Prophylaxis against venous thromboembolism in hospitalized medical patients: an evidence-based and practical approach. Pol Arch Med Wewn. 2008;118:209-215.

17. Dentali F, Douketis JD, Gianni M, et al. Anticoagulant prophylaxis to prevent symptomatic venous thromboembolism in hospitalized medical patients. Ann Intern Med. 2007;146:278-288.

18. Dobesh PP, Stacy ZA. Effects of a clinical pharmacy education program on improvement in the quantity and quality of venous thromboembolism prophylaxis for medically ill patients. JManag Care Pharm. 2005;11:755-762.

19. Stinnett JM, Pendleton R, Skordos L, et al. Venous thromboembolism prophylaxis in medically ill patients and the development of strategies to improve prophylaxis rates. Am J Hematol. 2005;78:167-172.

20. Kucher N, Koo S, Quiroz R, et al. Electronic alerts to prevent venous thromboembolism among hospitalized patients. $N$ Engl J Med. 2005;352:969-977.

21. Labarere J, Bosson JL, Brion JP, et al. Validation of a clinical guideline on prevention of venous thromboembolism in medical inpatients: a before-and-after study with systematic ultrasound examination. J Intern Med. 2004;256:338-348.

22. Scaglione L, Piobbici M, Pagano E, Ballini L, Tamponi G, Ciccone G. Implementing guidelines for venous thromboembolism prophylaxis in a large Italian teaching hospital:lights and shadows. Hematologica. 2005;90:678-684
Vascular Health and Risk Management

\section{Publish your work in this journal}

Vascular Health and Risk Management is an international, peerreviewed journal of therapeutics and risk management, focusing on concise rapid reporting of clinical studies on the processes involved in the maintenance of vascular health; the monitoring, prevention and treatment of vascular disease and its sequelae; and the involvement of

\section{Dovepress}

metabolic disorders, particularly diabetes. This journal is indexed on PubMed Central and MedLine. The manuscript management system is completely online and includes a very quick and fair peer-review system, which is all easy to use. Visit http://www.dovepress.com/ testimonials.php to read real quotes from published authors. 